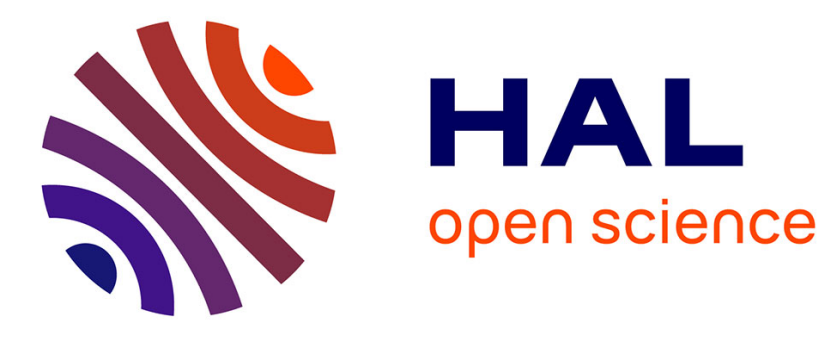

\title{
Computational Biomechanics of the Brain in the Operating Theatre
}

Hadrien Courtecuisse, Fanny Morin, Ingerid Reinertsen, Yohan Payan, Matthieu Chabanas

\section{- To cite this version:}

Hadrien Courtecuisse, Fanny Morin, Ingerid Reinertsen, Yohan Payan, Matthieu Chabanas. Computational Biomechanics of the Brain in the Operating Theatre. Biomechanics of the Brain, Springer, pp.321-344, 2019, 978-3-030-04995-9. 10.1007/978-3-030-04996-6_13 . hal-02385041

\section{HAL Id: hal-02385041 https://hal.science/hal-02385041}

Submitted on 2 Dec 2019

HAL is a multi-disciplinary open access archive for the deposit and dissemination of scientific research documents, whether they are published or not. The documents may come from teaching and research institutions in France or abroad, or from public or private research centers.
L'archive ouverte pluridisciplinaire HAL, est destinée au dépôt et à la diffusion de documents scientifiques de niveau recherche, publiés ou non, émanant des établissements d'enseignement et de recherche français ou étrangers, des laboratoires publics ou privés. 


\title{
Computational biomechanics of the brain in the operating theater
}

\author{
Hadrien Courtecuisse ${ }^{1}$, Fanny Morin ${ }^{1,2}$, Ingerid Reinertsen ${ }^{3}$, Yohan \\ Payan $^{2}$, and Matthieu Chabanas ${ }^{2}$ \\ ${ }^{1}$ ICube, Strasbourg University, CNRS, France \\ ${ }^{2}$ Univ. Grenoble Alpes, CNRS, Grenoble-INP, TIMC-IMAG, \\ F-38000 Grenoble, France \\ ${ }^{3}$ SINTEF, Department of Medical Technology, Trondheim \\ NO-7465, Norway
}

\section{Introduction}

Surgical resection of a brain tumor is a precise and delicate procedure. It requires to remove the entire tumor, in order to prevent recurrence, while preserving as much as possible of surrounding healthy or eloquent tissues, to reduce the probability of functional disorders. However, tumors borders, especially when located deep into the parenchyma, are usually not clearly visible among healthy tissues. In addition, important displacements and deformations may occur between pre-operative images used for diagnosis and intra-operative configurations, which raises significant difficulties for the treatment.

The brain-shift corresponds to an intra-operative deformation of soft tissues, affecting the localization of internal structures of the brain. Multiple factors can impact the amplitude and direction of this non-linear deformation: the loss of cerebrospinal fluid (CSF), the positioning of the patient during surgery, the size of the craniectomy, the opening of the dura and the actions of the surgeon such as retraction or tissue resection [1]. Several studies $[2,3,4,5]$ have reported cortical surface and subcortical displacements larger than $20 \mathrm{~mm}$ and $7 \mathrm{~mm}$, respectively, that must be taken into account given the fact that the desired accuracy of tumor resection is generally below $2 \mathrm{~mm}$.

While neuronavigation has become standard in neurosurgery, intraoperative imaging has proven to be useful in many situations [6] and should be of growing importance in future years. Among intraoperative imaging modalities, Ultrasound (US) is a common because it can easily be integrated in the clinical workflow, and is widely available in medical centers. However, US images are of limited quality, entailing significant difficulties to identify deep internal 
structures. Contrary to ultrasound, intra-operative Magnetic Resonance Imaging (iMRI) is a mean of obtaining detailed images of patient's brain during the surgery. However, such installation is expensive and the acquisition of MRI images during surgery is a complex process that significantly increases the duration of the procedure.

To fill the gap between comprehensive pre-operative data and sparse, incomplete or low-quality intra-operative images, the general trend is to develop non-rigid registration method. This enables to visualize inner structures, surgical targets or planning at key moments of the surgery where assistance is needed. Many image-to-image registration algorithms were proposed in the literature $[1,7]$. In this paper, we will only focus on biomechanical model-based solutions. One of their main advantages is the ease to define the mechanical actions at the origin of tissue deformation as well as the boundary conditions and contacts between structures. In addition, it has proven to be consistent and robust when dealing with (very) sparse intra-operative data.

This article is organized as follows. In section 2 we will introduce the main steps and criteria of model-based registration approaches. Section 3 is dedicated to the presentation of our method [8], which relies on Finite Element modeling and vessels-based constraints from intra-operative US. We then provide detailed discussion and comparison regarding the criteria introduced previously as well as the constraints in the operating theater.

\section{Key steps for computational modeling in the operating theater}

This section summarizes key steps and modeling choices that could or should be taken into account before and during surgery. In the following section we will position our retrospective study [8] with respect to these criteria, and highlight the necessary steps that are still missing for intra-operative usage of the method.

\begin{tabular}{l|l} 
Before surgery & During surgery \\
\hline - Construct model geometry & - Acquire intra-operative images \\
- Define constitutive law & - Evaluate additional boundary condi- \\
- Set mechanical properties & tions and loads \\
- Define boundary conditions & - Use efficient computational method \\
- Perform pre-computations & - Model tissue-resection \\
& - Render the information
\end{tabular}

Table 1: Key steps for computational modeling in the operating theater. Terms in regular font are common to the majority of the models, while ones in italic could be considered for improvement. 


\subsection{Before surgery: generation of a patient-specific model}

For applications in the operating theater, any generic model of the brain is of little value and a patient-specific model must be built. This specificity first concerns the morphology of the parenchyma, the bulk of the model, and inner structures such as the dura mater, ventricles or tumor, if any. While the constitutive law of brain tissue is usually generic, estimating the patient's own rheological parameters could be a valuable addition, depending on the type of loads applied to the model. Finally, some boundary conditions, e.g. related to fixed or contacts areas, can already be formulated.

\subsubsection{Geometry}

The generation of a patient model commonly rely on pre-operative Magnetic Resonance Images (MRI) solely, with different sequences available (such as T1weighted with or without Gadolinium, T2 FLAIR, MR Angiography or Diffusion Tensor Imaging). After a diagnostic scan, another MRI is commonly acquired a day or a few hours before surgery, to provide up-to-date images to the neuronavigation system. Ideally, the model should be generated from this pre-surgery exam. Therefore, to be compatible with the clinical workflow, it is mandatory for the model generation process to be as automatic and robust as possible. Although many approaches have been proposed in the literature, the automatic generation of patient-specific models remains one of the major bottlenecks for the widespread use of any computational model in the operating room.

A first step is usually the segmentation of the brain from MRI. A comprehensive review of this widely studied topic is well beyond the scope of this chapter. To limit their complexity, computational models in the operating room are generally relatively coarse and do not detail the cerebral convolutions and rarely the gray and white matter layers. Therefore, automatic methods to segment all the parenchyma, for example the Brain Extraction Tool (BET) proposed by [9], could be well adapted. After segmentation, most of the existing works rely on a 3D Finite Element mesh of the domain. While hexahedral elements are preferred numerically, tetrahedrons are often used for meshing simplicity.

\subsubsection{Constitutive law}

Brain constitutive behavior and brain modeling have been extensively described in the previous chapters of this book. The behavior of brain tissues is widely admitted as non-linear, inhomogeneous and patient-specific. The range of modeling strategies is, however, very large, with constitutive laws from linear to hyperelastic or bi-phasic materials. For a use in the operating room, one of the major constraints is computational efficiency. Therefore, a trade-off must be found between a fine modeling and the practicability of the model. Any simulation requiring more than a few minutes could obviously not be compatible with clinic. 


\subsubsection{Mechanical properties}

To our knowledge, virtually all the existing models use generic mechanical properties reported in the literature from experimental measurements. With some exception, brain tissue stiffness is usually considered in the 1-10 $\mathrm{kPa}$ range (in equivalent Young's modulus, for the non-linear materials), with a Poisson ratio from 0.4 to 0.49 [10]. Tumor tissues are often modeled as stiffer, following clinical recommendations but without clear supporting data.

Although these orders of magnitude of the mechanical parameters are adequate, they cannot represent the variability among patients and their specificity and inhomogeneity. Therefore, assessing patient-specific mechanical properties, for example with MR Elastography [11], could be a valuable addition. While this may not be crucial when using imposed displacements [12], patient-specific values could improve the simulation accuracy when other types of loads are used (especially forces).

\subsubsection{Known boundary conditions}

Some boundary conditions can be determined pre-operatively, typically at the interface between the brain and dura mater. Depending on the procedure, inner structures may also be considered as fixed during surgery, such as the brain stem region, the tentorium cerebelli, ventricles or even the falx cerebri.

In most cases, the segmentation of these structures from MRI images is manual or semi-automatic. However, some authors have proposed automatic methods to fix the cerebellum [13] or simulate interactions with the cerebral falx and tentorium cerebelli [14]. Once segmented, these structures are used to fix nodes or to set up sliding contact boundary conditions.

\subsubsection{Pre-computations}

Whenever possible, pre-computations could be performed pre-operatively to speed up the resolution during surgery. Pre-computations can be purely numerical. When using a linear elastic model, a common approach is to compute and store the deformations resulting from elementary nodal displacements [15]. Other strategies model the non linearity of the brain through pre-computations $[16,17,18]$. The overall model response to a set of imposed nodal displacements then relies on the principle of superposition, or linear combination of pre-computed deformations. While pre-computations could last several hours, the intra-operative inverse problem is generally straightforward. Finally, numerical optimizations are also possible, for example by pre-inversing the stiffness matrix to handle contacts with Lagrange Multipliers [8]. 


\subsection{In the operating theater}

\subsubsection{Intra-operative imaging}

Numerous factors can change between the pre-operative planning and the surgery, such as the patient positioning, location and size of the craniotomy, loss of cerebrospinal fluid, anesthesia control or tissue retraction and resection. All these factors directly affect how the brain tissues deform. Therefore, simulations relying only on a pre-operative model and planning cannot be considered to provide reliable results. It is then mandatory to capture information, in the operating room, about the current patient tissue configuration and the realized procedure, to specify adequate loads and boundary conditions of the model. This new information is usually acquired at least once, typically after dural opening for craniotomy-induced brain-shift compensation, or at the end of a resection procedure to ensure all tumor tissues were removed. Whenever possible, repeated acquisitions could be used to track the deformations all along the procedure.

Many intra-operative imaging modalities have been used in neurosurgery [6], such as MRI (iMRI), Ultrasound (iUS), optical systems such as stereo vision CCD images or Laser Range Systems (LRS), and to a less extent intra-operative X-Rays system like iCT or Cone Beam CT. A review of the advantages and drawbacks of these imaging modalities can be found in [1].

\subsubsection{Processing intra-operative images}

Intra-operative imaging can be used to capture information about the patient during surgery. The acquired images must then be processed to extract relevant features that will be later transferred to the model as boundary conditions or loads. Examples of structures to segment are points on the exposed cortical surface, contours (e.g. the cortical or ventricles surface on iMRI), homologous landmarks or $3 \mathrm{D}$ vessels. Ideally, segmenting the intra-operative images should be fully automatic. However, semi-automatic methods are commonly used as long as interactions remain very limited.

\subsubsection{Intra-operative boundary conditions and loads}

Many authors have used imposed displacements on FE nodes to match the model with the structures segmented from intra-operative images. In this case, the computational model could be mostly considered as a representation of the warping function in a registration problem. The brain-shift phenomenon is not simulated directly, instead the model is used to extrapolate the deformations of the brain from a sparse set of data, on a mechanical basis.

As opposed to this approach, several authors have proposed to actually model the influence of gravity, loss of CSF of tissue resection on the brain, to simulate its deformation $[13,19]$. In that context, the collected intra-operative data are not derived as loads for a forward simulation, but are used to find the parameters leading to the optimal solution of an inverse problem. 


\subsubsection{Efficient computational methods}

Computational efficiency is a crucial factor in the operating room. While the entire process must be considered, including images acquisition and processing, the computational model resolution itself can be one of the main limiting criterion. Real-time or interactive simulation is generally not required in a neurosurgical context, but overall computation time should be limited to a couple of minutes. This additional duration could be especially acceptable if the surgery can be resumed just after data acquisition, while the model is ran and the pre-operative MRI images are updated.

\subsubsection{Modeling tissue resection}

Neurosurgery often requires the retraction and resection of brain tissue, for instance to remove a tumor, entailing many additional issues. Topological modifications (such as re-meshing or elements suppression) must then be performed on the model. These modifications invalidate any pre-computations, raising additional concerns about the computation time. An iterative approach is for example proposed in [20], involving several simulations and re-meshing steps providing updated images in approximately $7 \mathrm{mn}$. However, from the best of our knowledge, a functional, robust and validated solution has not yet been proposed to address the problem of resection while meeting the constraints of the operating room.

\subsubsection{Rendering the information}

Once the deformed configuration of the brain has been computed, this information must be rendered to the surgeon in a clear and informative way. The most straightforward solution is to use the deformation field derived from the brain shift modeling to map the pre-operative MRI image to the patient's brain during surgery. To go further, several groups have proposed augmented reality visualization, especially in the surgical microscope, to help fusing, understanding and visualizing complex medical imaging data and anatomical structures [21].

\subsection{Validation and clinical studies}

Validating the fidelity of a computational model of the brain in itself, from a mechanical point of view, is a difficult problem. In a surgical context, a more relevant objective may be to evaluate the overall procedure, i.e. the outcomes of the simulation with respect to actual data about the deformed tissues. Ideally, validation images to measure a target registration error should be acquired after resection, and should be different than the ones used to express the loads and boundary conditions.

To our knowledge, a comprehensive study including independent input data, acquired in real surgical conditions, and an independent post-resection iMRI, acquired for validation only, is not yet available. However, several groups have started acquiring such data, and results are expected soon. While the iMRI 
setup will influence the measured deformation, due to its length, removal of tools, tissue debulking and dural closing, these images are expected to provide a much better ground truth than any other modalities.

\subsubsection{Practicability and integration in the surgical workflow}

Few studies clearly evaluate the practicability of their method. Several factors should be considered such as integration in the surgical workflow, especially to acquire intra-operative images, manual interactions, overall computation times and information rendering. In [8], our method required less than 2 min to process the iUS images, simulate the brain deformations with a constraint-based iterative method, then update the pre-operative MRI (only the iUS acquisition time is not included, usually 2 to 3 additional minutes). This method was evaluated in a retrospective study only, but we showed that both the hardware and the software could be compatible with the constraints of the operating room.

To our knowledge, almost all existing studies, including our own, are retrospective only. A notable exception is [22], in which simulations were carried out in the operating room, using the method proposed in [19], before updated MRI was displayed in the neuronavigation system. This study could then be considered as one of the first computational models of the brain actually used in the operating theater.

\section{Example of clinical application: constraint- based simulation during tumor resection}

This section introduces our approach to compensate for the brain-shift induced by the dura mater opening [8]. The method combines a biomechanical model build from pre-operative images and image-based constraints extracted from intra-operative US.

Before surgery, a patient-specific Finite Element (FE) model of the brain is constructed from pre-operative MRI images. This model accounts for the soft tissues morphology, but also vessels located around the tumor. During surgery, after opening the skull and dura mater, localized Doppler and B-mode ultrasound images were acquired directly in contact with the brain. The vascular tree and the footprint of the ultrasound probe are then extracted from these intra-operative images.

A biomechanical simulation is then performed to compensate for the brainshift deformation. We introduce several types of constraints allowing to i) model contacts between the brain and the dura, ii) register the pre- and intra-operative vessels and iii) constrain the cortical surface under the footprint of the probe. Finally, the pre-operative MRI is updated using the displacement field calculated from the biomechanical model An overview of this method is shown in Figure 1. 


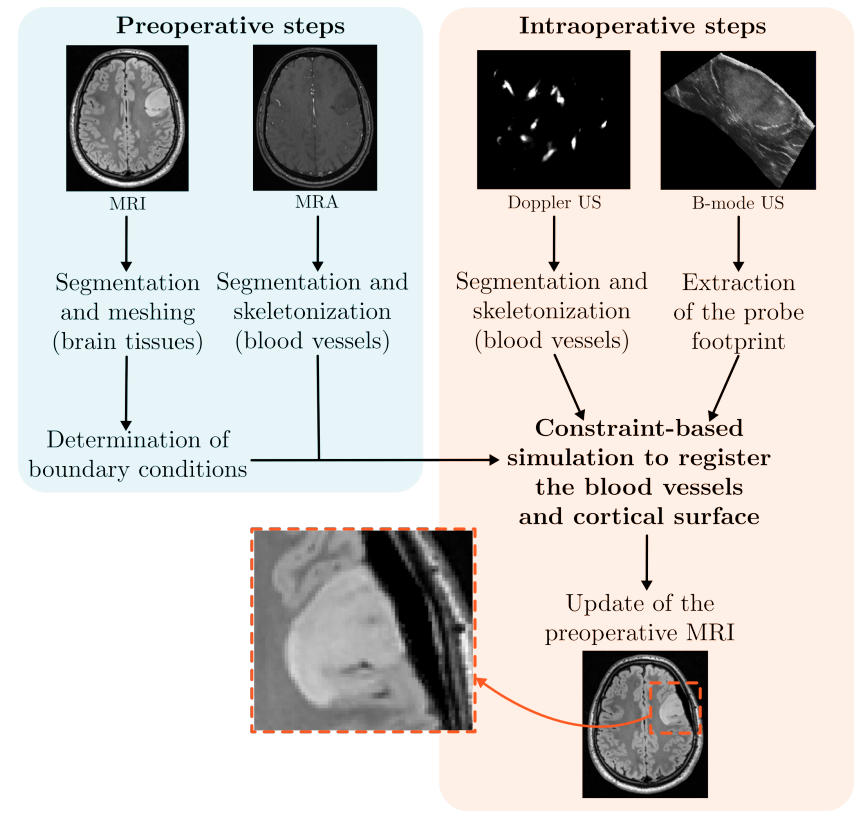

Figure 1: Overview of the method (source: [8]).

\subsection{Before surgery: model generation}

As presented in Section 2.1, the model must be built from pre-operative MRI images acquired a few hours before surgery. This procedure must then be as automatic as possible to be compatible with a clinical workflow.

\subsubsection{Geometries from MRI images}

Several atlas-based automatic algorithms such as [23, 24, 25] have provided detailed solutions, with regard to the resolution of the meshes, but their implementation is difficult. In our research context, we have chosen a semi-automatic coarse segmentation method.

Soft tissues are segmented from pre-operative T2-FLAIR (Fluid Attenuated Inversion Recovery) MRI. This sequence reduces the signal from the cerebrospinal fluid, thus highlighting brain lesions. The complete organ is first extracted using the BET (Brain Extraction Tool) algorithm proposed by [9]. It is fully automatic and runs in just a few seconds. The tumor is then segmented, after manually initializing some seeds, via a growing region algorithm implemented in the ITK-SNAP software [26]. For a more comprehensive study using our model in clinic, more advanced segmentation methods, potentially publicly available, should obviously be investigated.

Soft tissues are meshed homogeneously with linear tetrahedral elements, using the CGAL library [27], leading to a coarse mesh of 2000 nodes for a single 
hemisphere. Its surface is extracted to form the collision mesh that will be used to impose constraints with the skull and the probe footprint. This collision mesh is decimated (i.e. about 300 vertices) in order to reduce the number of constraints.

\subsubsection{Segmentation of the vascular tree}

The cerebrovascular tree is segmented from pre-operative Angiographic MRI (ARM). While this modality provides poor contrasts for soft tissues, vessels appear with very high intensities.

A review of vessels segmentation methods from medical images can be found in [28]. In our work, we reconstructed the cerebrovascular tree using the Maximal Intensity Projection (MIP) technique proposed by [29]. The histogram of this image reveals two peaks corresponding to the background of the image (black) and the soft tissues of the brain (dark gray), respectively. The vessels are then segmented using a threshold value chosen just after the second peak of the histogram. This is illustrated in Figure 2a.

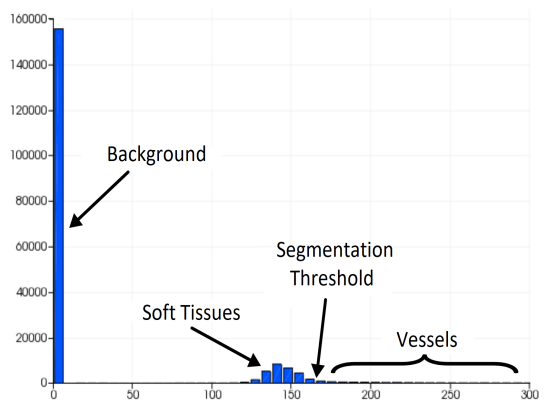

(a) Histogram of the MIP image

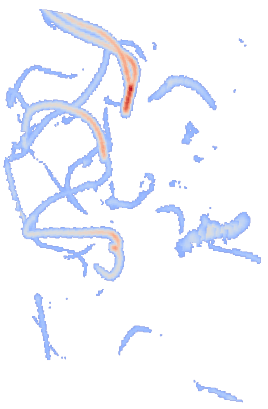

(b) Segmentation

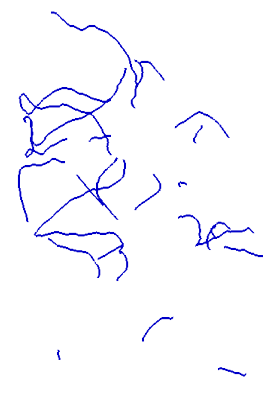

(c) Skeletization

Figure 2: Histogram of the MIP image, segmentation and mesh generation of the vessel tree.

The shape of this segmentation, and more particularly its outer envelope, is very sensitive to the image quality and segmentation parameters. For example, vessels often appear thicker in US than in ARM. In order to minimize the segmentation errors, center lines are extracted to form a skeleton passing through the center of the vessels. A modified Dijkstra algorithm is then employed where the Euclidian distance to the nearest wall is calculated for each voxel inside a vessel. Using the inverse of this distance as weights, images are then converted into a connected graph. Additional criteria are used to remove small branches that may appear as a result of a noisy segmentation (see [8] for details).

\subsubsection{Mechanical Coupling}

Several representations are used to describe the pre-operative biomechanical model. The brain is meshed and simulated with the Finite Element (FE) 
method, whereas constraints forces are applied on collision models (i.e. the surface and the vessels). Displacements must therefore be transferred from the FE mesh to the collision models, and conversely constraint forces must be integrated in $\mathrm{FE}$ equations. For this purpose, let $\mathcal{J}$ be a function providing the position of each vertex of the collision meshes $\mathbf{u}_{c o l}$ with respect to the nodal positions of the tetrahedral mesh $\mathbf{u}_{F E}$ :

$$
\mathbf{u}_{c o l}=\mathcal{J}\left(\mathbf{u}_{F E}\right)
$$

At the beginning of the simulation, the barycentric coordinates of each vertex of the collision models are associated with their closest tetrahedral element [30]. This association remains constant throughout the simulation, which can mathematically be written as a Jacobian matrix, defined by $\mathbf{J}=\partial \mathbf{u}_{c o l} / \partial \mathbf{u}_{F E}$. This Jacobian matrix is used to obtain the positions $\mathbf{u}_{c o l}$ from the $\mathbf{u}_{F E}$ positions. The previous equation can be rewritten as follows:

$$
\mathbf{u}_{c o l}=\mathbf{J} \mathbf{u}_{F E}
$$

In the opposite, constraint forces $\boldsymbol{\lambda}_{c o l}$ applied on the collision models are weighted with the same barycentric coefficients and converted in equivalent nodal forces on the finite element mesh $\boldsymbol{\lambda}_{F E}$ :

$$
\boldsymbol{\lambda}_{F E}=\mathbf{J}^{T} \boldsymbol{\lambda}_{c o l}
$$

\subsection{Intra-operative datasets}

Intra-operatively, registration data are extracted from the navigated ultrasound images. Power Doppler and B-mode signals are recorded simultaneously and the corresponding 3D image volumes are reconstructed. In a clinical context, the set of algorithms presented in the following paragraphs will have to be performed during the surgical procedure. They must therefore be fast, a few seconds, and almost fully automatic to allow their use in a clinical process.

\subsubsection{Vascular tree from Power Doppler ultrasound images}

Power Doppler ultrasound images provide a real-time visualization of flows, particularly the blood flow, offering a strong contrast of the vessels (see Fig. 3a). The intra-operative vascular tree is then segmented from these images by a thresholding method, using parameters tuned manually [31]. In addition, to limit the sensitivity of the method with respect to segmentation parameters, the skeletonization of the data is performed following the algorithm previously described for the ARM. Finally, in order to maintain computation time compatible with clinical constraints and to avoid over-constrained problems, the vascular tree model is sampled with 1 point every $2.5 \mathrm{~mm}$ (this value could adapted to the mesh resolution). 


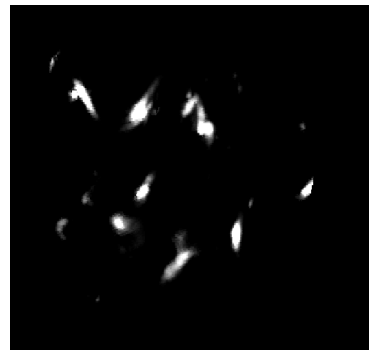

(a) Doppler Image

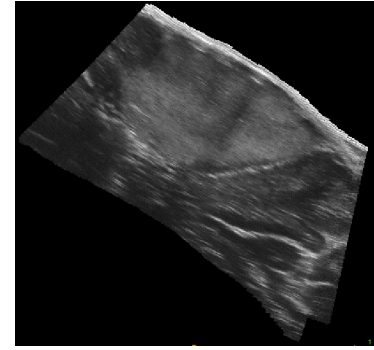

(b) B mode image

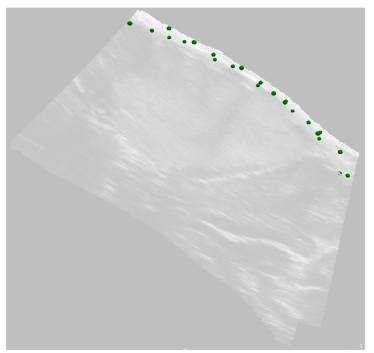

(c) Point cloud generation

Figure 3: Extraction of the probe footprint in US B mode images.

\subsubsection{Probe footprint from B-mode ultrasound images}

The ultrasound probe footprint located at the interface between the probe and brain tissues is easily identifiable, and can thus be extracted from B mode images in order to provide additional constraints at the surface of the brain. The visualization of this boundary arises from an ultrasound artifact: when the waves enter the tissues of the brain, a part is reflected on the surface forming this specific band in the image (see Figure $3 \mathrm{~b}$ ). The footprint being located on the edges of the images, the outer envelope of the volume is extracted. A low-pass filter is then applied to provide a binary volume, and finally a point cloud is generated sampling data at 1 point every $10 \mathrm{~mm}$ (see Fig. 3c).

\subsection{Biomechanical model: formulation}

In the context of registration with intra-operative data, a static problem can be considered. The governing equation is given by:

$$
\mathcal{F}\left(\mathbf{u}_{F E}\right)+\mathcal{H}\left(\mathbf{u}_{c o l}, \mathbf{p}_{\mathcal{I}}\right) \boldsymbol{\lambda}=\mathbf{0}
$$

where $\mathcal{F}\left(\mathbf{u}_{F E}\right)$ is a nonlinear function describing the internal forces according to the displacement $\mathbf{u}_{F E}$ of the FE model. $\mathcal{H}\left(\mathbf{u}_{c o l}, \mathbf{p}_{\mathcal{I}}\right)$ is a nonlinear function associating Lagrangian Multipliers $\boldsymbol{\lambda}$ used to impose constraint forces to the FE model. The number and directions of Lagrangian Multipliers depend on the collision models positions, $\mathbf{u}_{c o l}$, and the positions extracted from intra-operative images, $\mathbf{p}_{\mathcal{I}}$. In addition, the solution $\mathbf{u}_{F E}$ to this problem must satisfy a set of constraints equations which can mathematically be represented as follows:

$$
\mathcal{H}\left(\mathbf{u}_{c o l}, \mathbf{p}_{\mathcal{I}}\right)=\boldsymbol{\delta}
$$

where $\boldsymbol{\delta}$ is the violation of the constraints (for example penetration through a boundary surface).

\subsubsection{Constitutive law and parameters}

As seen in Section 2.1.2, the constitutive behavior of the brain is known to be complex and should be described using hyper-elastic laws. However, in our 
constraint-based context, displacements are imposed through Lagrangian Multipliers. The solution in displacements is thus weakly sensitive to the constitutive law and parameters $[32,12]$. A linear elastic law is therefore used. Following [33], the Young's modulus and Poisson's ratio are respectively set to $E=1.5$ $\mathrm{kPa}$ and $\nu=0.45$. A higher stiffness of $E=10 \mathrm{kPa}$ is used for the tumor. To take into account large displacements while being computationally efficient, simulations are run using the corotational approach [34].

\subsubsection{Constraints}

Two types of constraints are used during the registration process:

- Bilateral constraints $(\boldsymbol{\delta}=\mathbf{0})$ used to impose displacement such that constrained points of the model are moved to a desired position.

- Unilateral constraints $(\boldsymbol{\delta} \perp \boldsymbol{\lambda}$ i.e. $\boldsymbol{\delta} \geq \mathbf{0}$ and $\boldsymbol{\lambda} \geq \mathbf{0}$ and $\boldsymbol{\delta} \cdot \boldsymbol{\lambda}=\mathbf{0}$ ) are used to impose contacts between structures. In other words, either the violation is positive $(\boldsymbol{\delta} \geq \mathbf{0})$ and no contact forces are applied $(\boldsymbol{\lambda}=\mathbf{0})$ or the violation must be nullified $(\boldsymbol{\delta}=\mathbf{0})$ with a positive constraint force $(\boldsymbol{\lambda} \geq \mathbf{0})$.

Due to the non-linearity of $\mathcal{H}$, a unique solution does not exist. Instead, we use an iterative method where the problem is linearized at each iteration, allowing for the convergence towards the closest local minimum. Constraint equations are then defined at the beginning of each iteration using the current position of the model, and are assumed to be constant during the solving process $\mathcal{H}\left(\mathbf{u}_{c o l}, \mathbf{p}_{\mathcal{I}}\right) \simeq \partial \mathcal{H} / \partial \mathbf{u}=\mathbf{H}$. The constraint matrix $\mathbf{H}$ (known as the Jacobian of the constraints) contains the directions on which Lagrangian Multipliers are computed.

For each iteration $i$, a single iteration of a Newton-Raphson method is solved while constraint equations are defined following an Iterative Closest Point (ICP) algorithm [35]. Each point extracted from the intra-operative data is associated with its closest element on the biomechanical model (see section 3.4), defining this way the directions (stored in the matrix $\mathbf{H}^{i}$ ) in which constraint forces $\boldsymbol{\lambda}$ are applied to perform the registration. The linearized equation provides the following Karush-Kuhn-Tucker (KKT) system:

$$
\left\{\begin{array}{ccc}
\mathbf{K}^{i} \Delta \mathbf{u}^{i}+\mathbf{H}^{i^{T}} \boldsymbol{\lambda} & = & -\mathcal{F}\left(\mathbf{u}^{i}\right) \\
\mathbf{H}^{i} \Delta \mathbf{u}^{i} & = & \boldsymbol{\delta}
\end{array}\right.
$$

with $\mathbf{K}^{i}=\partial \mathcal{F} / \partial \mathbf{u}$ is the global stiffness matrix and $\Delta \mathbf{u}^{i}=\mathbf{u}^{i+1}-\mathbf{u}^{i}$ the difference in position between two iterations.

\subsubsection{Solving process}

The systems are solved with a schür complement method. This requires the computation of the Delassus operator $\mathbf{W}=\mathbf{H}^{i} \mathbf{K}^{i^{-1}} \mathbf{H}^{i^{T}}$ which is the most time-consuming operation. In our context, a large number of constraints needs 
to be applied on the FE model $\left(\mathbf{H}^{i}\right.$ is a sparse matrix whose dimension is the number of constraints times the number of degrees of freedom of the FE model). Moreover, even for a relatively coarse FE mesh, computing the inverse of $\mathbf{K}^{i-1}$ at each simulation step would be too expensive, preventing an intra-operative use of the method. Instead we used the Compliance warping method introduces by [36] where the Delassus operator is approximated using the stiffness matrix inverted at the initial step and nodal rotations: $\mathbf{W}=\mathbf{H}^{i} \mathbf{R}^{i} \mathbf{K}^{0^{-1}} \mathbf{R}^{i^{T}} \mathbf{H}^{i^{T}}$, where $\mathbf{R}^{i}$ is the current nodal rotation derived from the corotational formulation [34].

Finally a modified Gauss-Seidel solver provides $\boldsymbol{\lambda}$ satisfying the KKT conditions (see [37] for details). At equilibrium, i.e. $\Delta \mathbf{u}_{F E}=\mathbf{0}$, the displacement field of the FE model is used to warp the pre-operative MRI image.

\subsection{Constraint-based iterative registration}

Let $\mathcal{M}$ be the pre-operative model, $\mathcal{P}$ be the collision models (obtained with the barycentric mapping $\mathbf{J}$ ) and $\mathcal{I}$ the intra-operative data. Three types of constraints are used to match $\mathcal{M}$ to $\mathcal{I}$ :

Boundary conditions: Bilateral constraints are applied between the external surface of the brain model $\mathcal{M}$ and $\mathcal{P}_{\text {dura }}$. Tissue is then prevented from exiting the skull cavity, and can slide in contact with the dura mater. These constraints are removed in the craniotomy area, identified in the ultrasound B-mode images, so that tissues can sag. Finally, a few nodes are fixed in the cerebellum area.

Cortical surface: Unilateral constraints force $\mathcal{M}$ to remain under the US probe footprint $\mathcal{I}_{\text {probe }}$. However, tissue can sag and are not necessarily in contact.

Vessels registration: The pre- and intra-operative vascular trees $\mathcal{P}_{\text {vessels }}$ and $\mathcal{I}_{\text {vessels }}$ are registered using bilateral constraints.

All these constraints are integrated and solved with the same formalism, described in equation (6). At the end of each simulation step, a $\boldsymbol{\lambda}$ force is computed allowing to impose displacements on the model satisfying the constraints. Yet, a major difficulty of this constraint-based lies in the uncertainty during the pairings step. Indeed, although pre- and intra-operative data represent the same anatomical structures, their characteristics and quality may differ greatly due to noisy data and calibration errors, inherent in clinical applications. For this reason, we introduce the notion of sliding constraints. We voluntary under sample the constraints, allowing this way to partially solve the registration problem while letting the mechanical model deform and refine pairing of data

over the iterations. The overall iterative process is depicted in Figure 4 and in algorithm 1. 

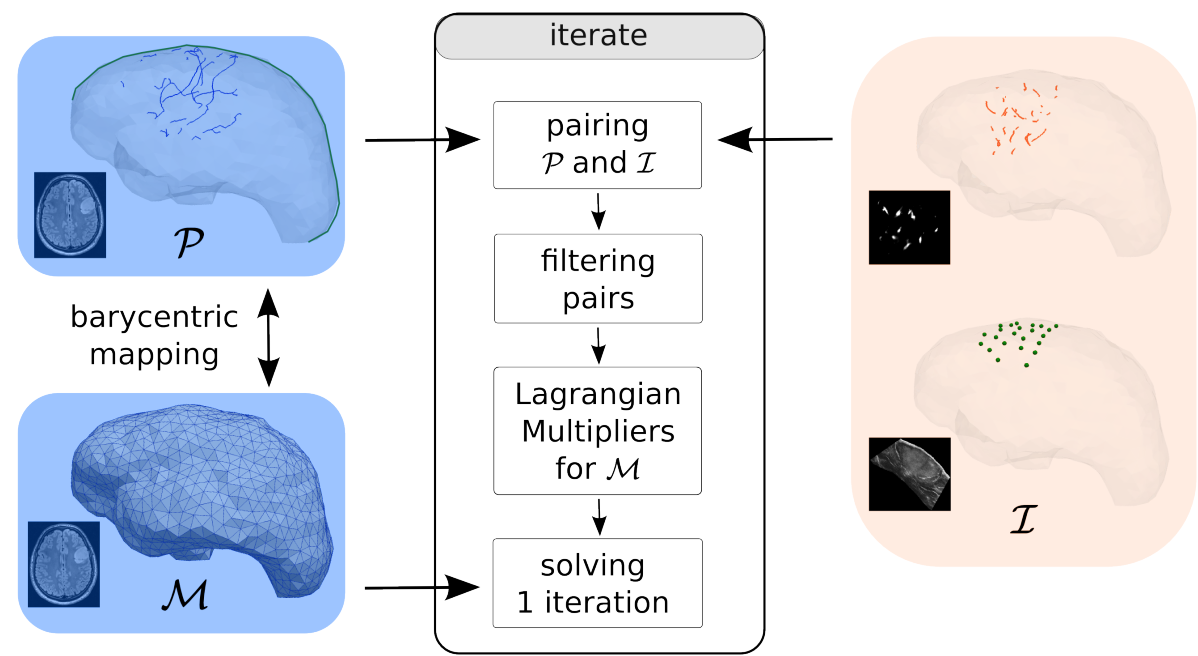

Figure 4: iterative registration process. Pairings between pre- and intraoperative datasets $\mathcal{P}$ and $\mathcal{I}$ are computed, then filtered. This defines the directions of the Lagrangian Multipliers constraints, stored in matrix $\mathbf{H}$, applied to the biomechanical model $\mathcal{M}$ so that it deforms towards $\mathcal{I}$.

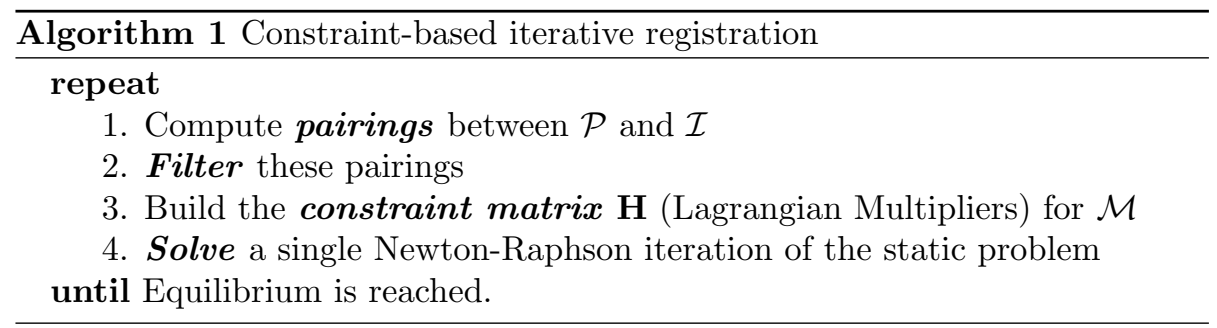




\subsubsection{Computing pairings}

The first step of each iteration consists of computing a set of pairings between $\mathcal{P}$ and $\mathcal{I}$, defining the distances to minimize. Depending on the structures and their representation, point-to-surface, point-to-segment or point-to-point projections can be used.

In a typical scenario the pre-operative dataset $\mathcal{P}$ is the best defined, using continuous curves or surfaces, while $\mathcal{I}$ can simply be a set of points. Just like in a standard Iterative Closest Point method (ICP), each point $q_{\mathcal{I}}$ of $\mathcal{I}$ is paired with its closest projection $q_{\mathcal{P}}$ on $\mathcal{P}$. Several directions $n_{j}$ are associated:

- when projecting on a plane, $n_{1}$ is the plane's normal on $q_{\mathcal{P}}$.

- when projecting on a line, $n_{1}$ is the direction of projection and $n_{2}$ is orthogonal to both $n_{1}$ and the line's tangent (see Figure 5).

- when projecting on a point, $n_{1}, n_{2}$ and $n_{3}$ form an orthonormal referential centered on $q_{\mathcal{P}}$.

While the pairing is usually identified from $\mathcal{I}$ to $\mathcal{P}$, the associated constraints are applied on $\mathcal{M}$, along directions $n_{j}$, so that it deforms towards $\mathcal{I}$.

\subsubsection{Filtering pairings}

A crucial step is to filter the pairings to remove aberrant ones. The main strategies are the following:

- when several vertices of $\mathcal{I}$ are projected on the same line segment or triangle, only the nearest one is kept [8].

- when a pair's distance is larger than the median distances plus a given threshold, or smaller than another threshold, this pair discarded [38, 8].

- when available, second degree information can also be taken into account in addition to Euclidian distances. For example, elements with a different orientation cannot be paired [37].

After this filtering, remaining pairings may also be subsample. First, this can limit the total number of constraints eventually applied on $\mathcal{M}$, to spare computation time. Also, it is important to avoid having multiple constraints, potentially antagonists, on single or neighbor FE elements. Therefore, pairings initially defined between $\mathcal{P}$ and $\mathcal{I}$ must be later sampled with respect to the $\mathcal{M}$ mesh refinement.

\subsubsection{Sliding Constraints}

While filtering allows to prune inconsistent pairings that may arise from noisy data, this criterion is not sufficient. Indeed, due to the deformation between pre- and intra-operative images some positions of $\mathcal{I}$ may have shifted to closer structures in the pre-operative model. However, this may not be the actual 
corresponding position of the point on the vessel, which remains unknown. For example, a point may be projected on a given vessel, but its position on this vessel is not exactly known. It is therefore important to not completely constrain the model and impose displacements in this direction; otherwise a point would always, at the end of the simulation, remain on its initial projection.

A main advantage of our method is the notion of sliding constraints. When a constraint is applied along a direction $n_{i}$, forces $\boldsymbol{\lambda}$ are only computed so that no violation of the constraint remains along that direction. However, the initial point can end up anywhere on the plane orthogonal to $n_{i}$, not necessarily on its initial projection. Note that bounding $\boldsymbol{\lambda}$ forces may break that condition, in case of very large (mis)pairing distances. The shift in the orthogonal direction is given by the constraint solver minimizing the energy necessary to satisfy the set of constraints.

Depending on the type of elements the sliding constraints are defined along the following directions:

- a projection on a plane yields to a single constraint along direction $n_{1}$. The point can slide along this plane: after resolution, it will be located on the plane, but not necessarily on its initial projection.

- a projection on a line segment yields two constraints along $n_{1}$ and $n_{2}$. The point can slide along this line: after resolution, it will be located on the line, but not necessarily on its initial projection.

An example of sliding constraint is given in Figure 5. For an iteration $k$ of the registration method, each point can slide along its segment of projections. Its final position depends on all other constraints applied to the model. The next iteration(s) will then enable the convergence towards a local solution.

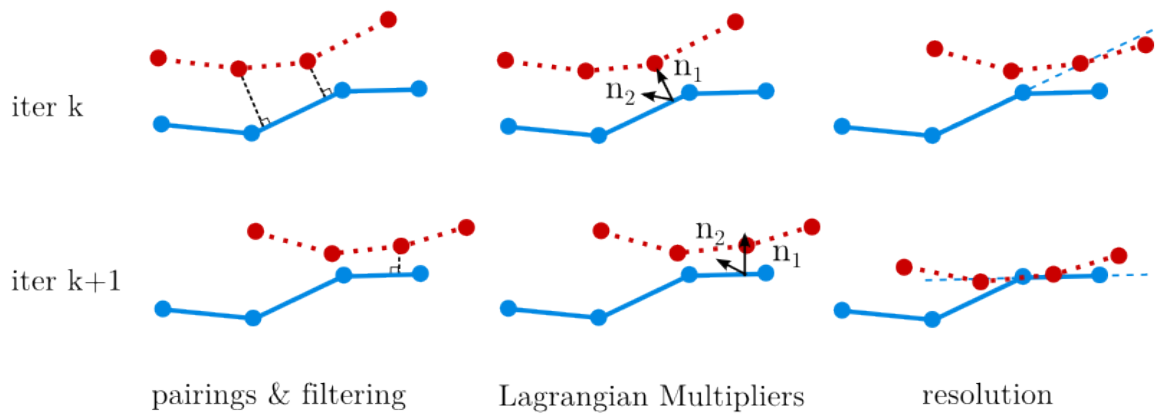

Figure 5: sliding constraints

Advantages of using the sliding constraints in an iterative registration process are then:

- no a priori information is required during the pairing step. Closest projections only are used, plus the filtering step. 
- in the case of mispairings, for example projection of a point onto the wrong vessel, the considered point will end up far from its initial target projection, if other better-suited constraints force the model into another direction.

- in the case of antagonist constraints, none or few of them should be resolved, and a new iteration will occur.

- at each new iteration $k$, pairings will be redefined. The expected behavior is that the uncertainty of the pairings will decrease as long as the process converges towards an overall acceptable solution.

\subsection{Clinical evaluation}

As presented in [8], our method has been evaluated on five retrospective cases. Data were collected by the SINTEF Medical Technology Institute at St. Olav University Hospital (Trondheim, Norway) [38]. For each clinical case, T2FLAIR and Angiographic MRI images were acquired before surgery. During the procedure, ultrasound images were acquired with the navigation system Sonowand [39]. These data were collected through a clinical study approved by the local ethics committees and the consent of each patient was obtained prior to the procedure.

\subsubsection{Quantitative and qualitative results after dural opening}

Our method has been evaluated and compared to the modified ICP (providing a rigid registration) of $[31,40,38]$, available in the open-source framework for image-guided therapy CustusX [41]. To obtain quantitative results, the first difficulty was to define reliable landmarks visible in both image modalities. For each case, 5 to 9 landmarks were first identified in blood vessels bifurcations, by two operators, on both the pre-operative MRA and intra-operative Power Doppler US images. These landmarks were used to define a target registration error (except for one patient for which the quality of US was not enough to define landmarks). In addition, anatomical structures such as sulcus were delineated by a clinician in the MRI and B-mode US acquisitions, allowing a measurement that is completely independent from the data used by the method. Note that the measured errors, with vessel landmarks and sulci, are sub-surface and located in the region of interest i.e. the tumor.

Figures $6 \mathrm{a}$ shows an example of simulation and $6 \mathrm{~b}$ and $6 \mathrm{c}$ the corresponding errors for 4 patients. On average $72 \%$ of the deformation occurring is corrected with our method, with a mean error under $1.5 \mathrm{~mm}$ for each patient [8]. In addition, less than 2 minutes are required to segment the intra-operative US images, run the simulation and update the MR images, which is compatible with a clinical use.

After registration, MRI is warped using the displacement field of the FE model (see Fig. 7, right). Qualitative observations, especially deep around the tumor, show consistent matching between the warped MRI and US images 
whereas significant errors can be observed in the initial pre-operative MRI, enhancing the importance of the deformation.

\subsubsection{Experiments during tumor resection}

After considering craniotomy-induced brain-shift after dural opening, our method has also been evaluated to compensate for deformations during resection [42]. For four of the five patients in the study, an intra-operative ultrasound scan was also acquired during tumor resection. Note that the method was used as it is on these images, without taking into account any topological modifications in the model, nor using any continuity from the first ultrasound acquisition.

For the first 3 patients, the mean target registration error measured on vessel landmarks is $1.76,2.35$ and $1.89 \mathrm{~mm}$, respectively. Distances on sulci are also globally improved, and qualitative results show a very satisfying adequation in the deep tissue surrounding the tumor (see Figure 8). Overall, $63 \%$ of the shift is compensated during resection, which is less than after dural opening but still a very good improvement.

These promising results have not been confirmed on the fourth patient, with a brain-shift reduced from 9 to $6.5 \mathrm{~mm}$ only. Two sources of error could explain the failure of this case. First, vessels are located on one side of the tumor only, which can explain the poor lateral accuracy. Mostly, this is a deep tumor while the first 3 cases were superficial ones. The amount of deformation is here much

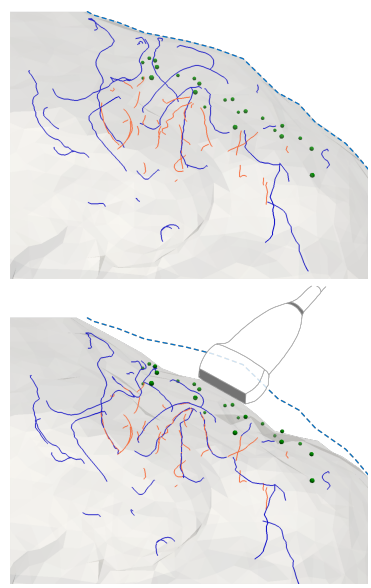

(a) Registration

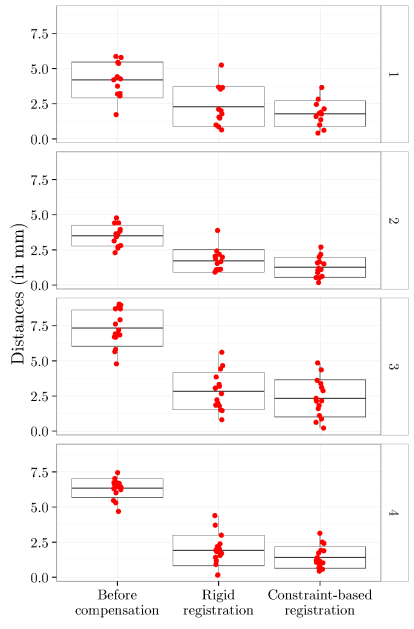

(b) Landmarks

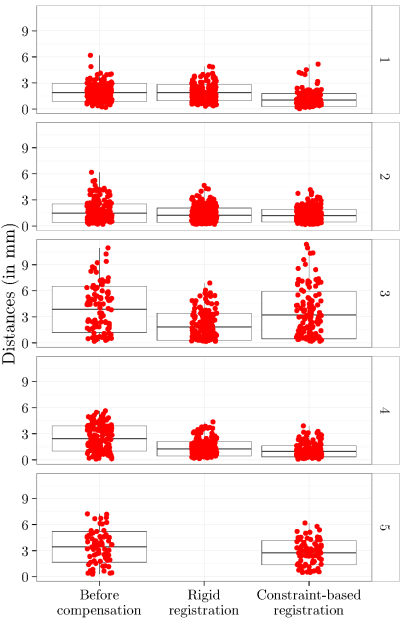

(c) Structures

Figure 6: Brain shift compensation using constraint-based simulation. Pre- and intra-operative blood vessels, respectively in blue and orange, are registered. Other constraints maintain the brain in sliding-contact with the dura matter and under the US probe. The Hausdorff distance between landmarks and delineated anatomical structures manually segmented are shown in graphs $6 \mathrm{~b}$ and $6 \mathrm{c}$. 


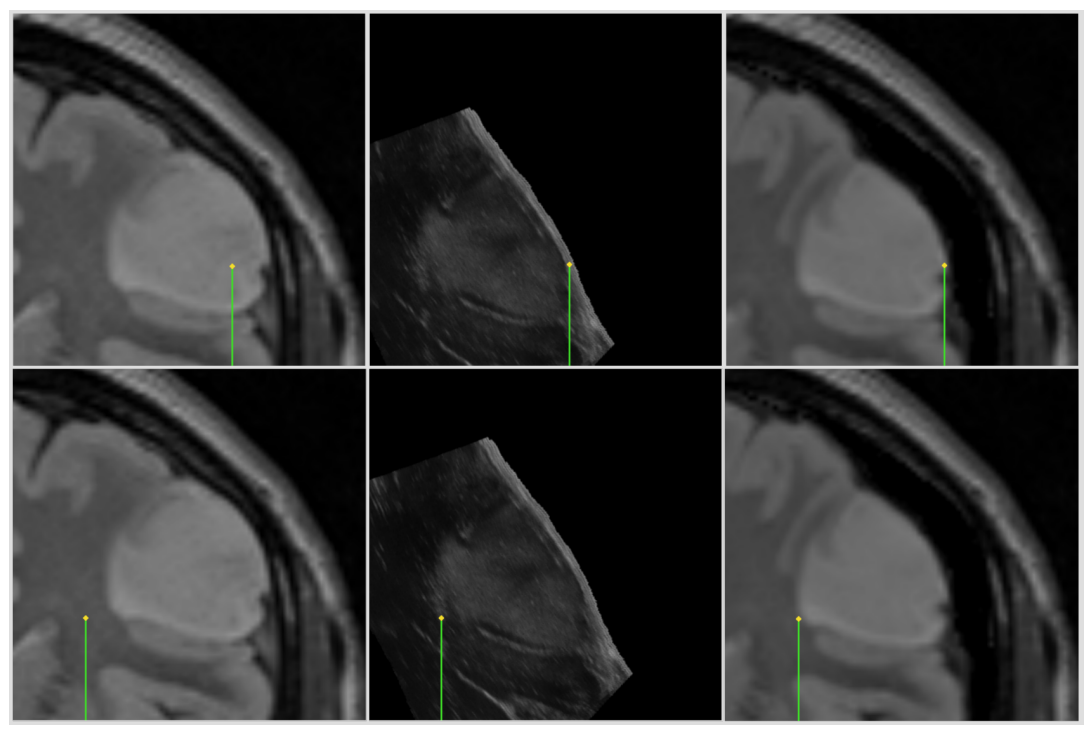

Figure 7: Example of pre-operative MRI, B-mode intra-operative US and MRI updated with our method. A pointer first shows the borders of the exposed cortical surface (top row) then a deep sulcus bifurcation point (bottom row).

larger and the retraction to create the surgical channel should clearly be taken into account.

While more cases must be considered, these first results have shown the ability of the method to compensate for resection-induced brain-shift, without additional treatments, in the case of a peripheral tumor. While deformation and topological changes occur locally within the tissues, reliable surrounding vessels still remain to register the pre- and intra-operative exams. However, more complex cases require additional treatment, especially the simulation of tissue resection in the biomechanical model.

\subsubsection{Practicability}

Although validated in a retrospective study only, our method should be usable in a clinical context. Indeed, it has been developed so that most of the steps, especially the intra-operative ones, are performed with very limited interactions of an operator and short computation times.

Pre-operative steps Segmentation of the pre-operative MRI remains one of the main limitations for a widespread use of our method in clinic. Indeed, while the whole brain is extracted via the automatic BET algorithm [9], the segmentation of the tumor and the other anatomical structures remains manual. Nevertheless, the segmentation of pre-operative images tends to become a standard step of nowadays clinical routines. Morevoer, several solutions exist 


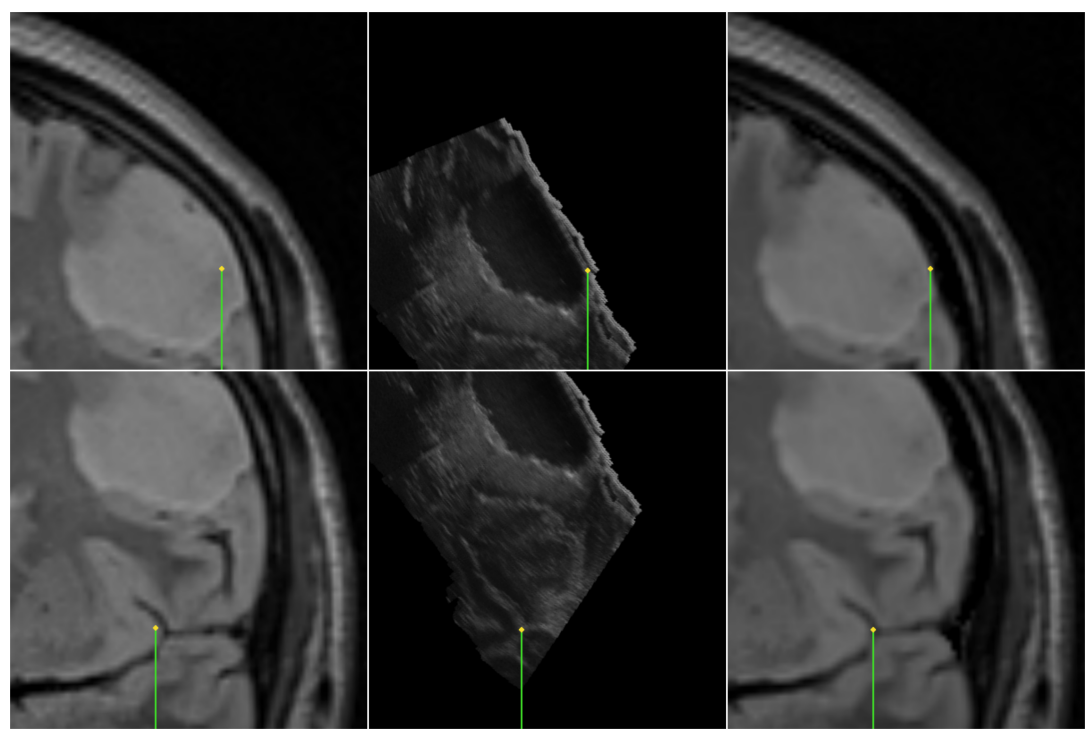

Figure 8: Brain-shift compensation during resection for patient 1 using the constraint-based biomechanical method. Slices of the pre-operative MRI, Bmode ultrasound image and updated MRI are shown. A pointer first shows the borders of the exposed cortical surface (top row) then a deep sulcus bifurcation point (bottom row).

in literature $[23,24,25]$ for automatic extraction of the brain and its various anatomical structures from MRIs.

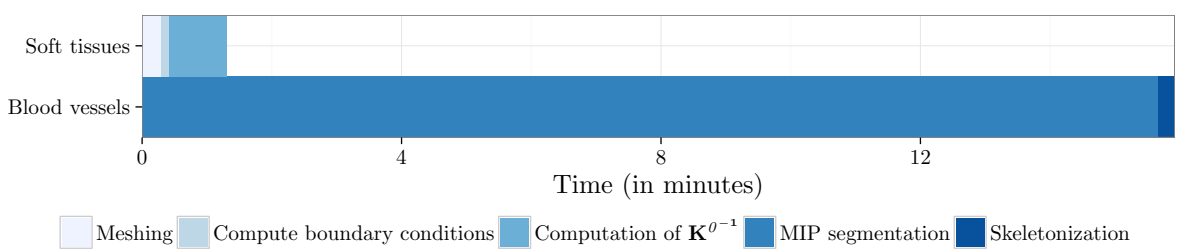

Figure 9: Execution time of the pre-operative steps (without taking into account the time required for segmentation).

The generation of pre-operative models was performed in approximately 20 $m n$ (after the segmentation of the brain). As shown in Figure 9, constructing the soft tissue model includes mesh generation (FE and collision meshes), definition of boundary conditions and inversion of the stiffness matrix (denoted $\mathbf{K}^{0^{-1}}$ ). Given the segmentation of the structures, all these steps are performed automatically. The most expensive part is the segmentation of the vessels in the ARM image and their skeletonization, which takes about sixteen minutes. Manual interactions are still needed, but as said before this is acceptable in the 
clinical workflow as these operations can be performed on the day before the surgery.

Intra-operative steps A $11 \mathrm{~L}$ linear probe attached to the Vivid E9 ultrasound scanner (GE Vingmed Ultrasound, Horten, Norway) was used for all acquisitions. The 3D reconstruction of images and the initial rigid transformation were performed intra-operatively with the CustusX framework, which was successfully used in a clinical workflow [38]. The additional time of our method is shown in Figure 10. Less than two minutes are required to provide updated images intra-operatively (approximately $30 \mathrm{sec}$ for the extraction of vessels and the footprint, 1 to $2 \mathrm{~mm}$ for the simulation and $10 \mathrm{sec}$ to warp the image). In addition, the mode $\mathrm{B}$ and Doppler ultrasound images being acquired simultaneously, the extraction of the footprint of the probe and the vessels can be performed in parallel.

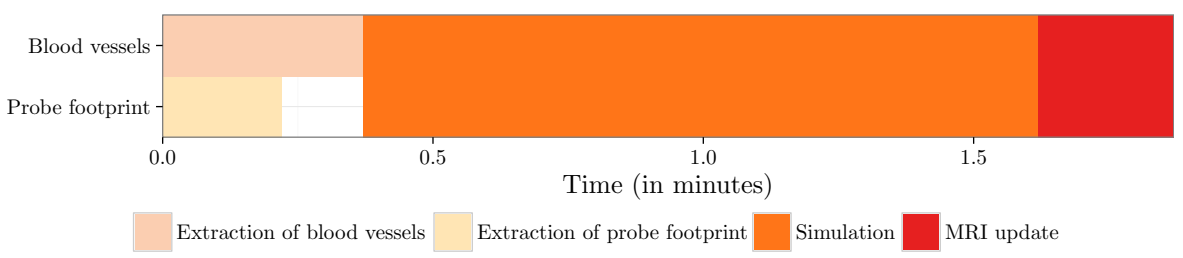

Figure 10: Execution time of the intra-operative stages, after acquisition of the ultrasound images and $3 \mathrm{D}$ reconstruction of the image volume.

Sixteen and two minutes are required for pre- and intra-operative steps, respectively. Given the fact that brain surgery generally lasts several hours, this overhead seems acceptable for a future use of our approach in the operative theater.

\section{Conclusion}

A model-based method was proposed to register pre- and intra-operative medical images. It can be seen as an ICP-like approach with a biomechanical model as the warping function, instead of a geometrical one, driven by Lagrangian Multipliers constraints. The method was successfully evaluated on five retrospective clinical cases, yielding to an efficient compensation of the brain shift. The method was also tested for resection-induced brain-shift compensation, and provided a significant improvement of the registration. Future works will focus on topological modifications of the FE model allowing for the simulation of the separation of resected tissues. A clinical study is also in preparation to actually use our computational biomechanical model of the brain in the operating theater. 


\section{Acknowledgment}

This work was partly supported by the French National Research Agency (ANR) through the frameworks Investissements dAvenir Labex CAMI (ANR-11-LABX0004) and Infrastructure dAvenir en Biologie et Santé (ANR-11-INBS-0006),

and by a France-Norway partnership (PHC Aurora 2015/Research Council of Norway).

\section{References}

[1] I. J. Gerard, M. Kersten-Oertel, K. Petrecca, D. Sirhan, J. A. Hall, and D. L. Collins, "Brain shift in neuronavigation of brain tumors: A review," Medical Image Analysis, vol. 35, pp. 403-420, 2017.

[2] D. L. G. Hill, C. R. Maurer Jr, R. J. Maciunas, J. A. Barwise, M. J. Fitzpatrick, and M. Y. Wang, "Measurement of intraoperative brain surface deformation under a craniotomy," Neurosurgery, vol. 43, no. 3, pp. 514-526, 1998.

[3] D. W. Roberts, A. Hartov, F. E. Kennedy, M. I. Miga, and K. D. Paulsen, "Intraoperative Brain Shift and Deformation: A Quantitative Analysis of Cortical Displacement in 28 Cases," Neurosurgery, vol. 43, no. 4, pp. 749 758, 1998.

[4] C. Nimsky, O. Ganslandt, S. Cerny, P. Hastreiter, G. Greiner, and R. Fahlbush, "Quantification of, Visualization of, and Compensation for Brain Shift Using Intraoperative Magnetic Resonance Imaging," Neurosurgery, vol. 47, no. 5, pp. 1070-1080, 2000.

[5] A. Nabavi, P. M. Black, D. T. Gering, C.-F. Westin, V. Mehta, R. S. Pergolizzi, M. Ferrant, S. K. Warfield, N. Hata, R. B. Schartz, W. M. Wells, R. Kikinis, and F. A. Jolesz, "Serial Intraoperative Magnetic Resonance Imaging of Brain Shift," Neurosurgery, vol. 48, no. 4, pp. 787-798, 2001.

[6] K. Narang, A. Jha, and M. Schulder, Intraoperative Imaging in Neurosurgery. Jaypee, The Health Sciences Publishers, 2017.

[7] S. Bayer, A. Maier, M. Ostermeier, and R. Fahrig, "Intraoperative Imaging Modalities and Compensation for Brain Shift in Tumor Resection Surgery," International Journal of Biomedical Imaging, vol. 2017, pp. 1-18, 2017.

[8] F. Morin, H. Courtecuisse, I. Reinertsen, F. Le Lann, O. Palombi, Y. Payan, and M. Chabanas, "Brain-shift compensation using intraoperative ultrasound and constraint-based biomechanical simulation," Medical Image Analysis, vol. 40, pp. 133-153, 2017.

[9] S. M. Smith, "Fast Robust Automated Brain Extraction," Human Brain Mapping, vol. 17, no. 3, pp. 143-155, 2002. 
[10] F. Morin, M. Chabanas, H. Courtecuisse, and Y. Payan, "Biomechanical modeling of brain soft tissues for medical applications," in Biomechanics of Living Organs: Hyperelastic Constitutive Laws for Finite Element Modeling, pp. 127-146, Elsevier, 2017.

[11] R. Muthupillai, D. J. Lomas, P. J. Rossman, J. F. Greenleaf, A. Manduca, and R. L. Ehman, "Magnetic resonance elastography by direct visualization of propagating acoustic strain waves," Science, vol. 269, no. 5232, pp. 18541857, 1995.

[12] K. Miller and J. Lu, "On the prospect of patient-specific biomechanics without patient-specific properties of tissues," Journal of the mechanical behavior of biomedical materials, vol. 27, pp. 154-166, 2013.

[13] P. Dumpuri, R. C. Thompson, B. M. Dawant, A. Cao, and M. I. Miga, "An atlas-based method to compensate for brain shift: Preliminary results," Medical Image Analysis, vol. 11, pp. 128-145, 2007.

[14] I. Chen, A. M. Coffey, S. Ding, P. Dumpuri, B. M. Dawant, R. C. Thompson, and M. I. Miga, "Intraoperative Brain Shift Compensation: Accounting for Dural Septa," IEEE Transcations on Biomedical Engineering, vol. 58, no. 3, pp. $499-508,2011$.

[15] M. Bucki, O. Palombi, M. Bailet, and Y. Payan, "Doppler Ultrasound Driven Biomechanical Model of the Brain for Intraoperative Brain-Shift Compensation: A Proof of Concept in Clinical Conditions," in Soft Tissue Biomechanical Modeling for Computer Assisted Surgery, pp. 135-165, Springer, 2012.

[16] F. Chinesta, A. Huerta, R. G., and K. Willcox, "Model reduction methods," in Encyclopedia of Computational Mechanics (2nd ed.), Wiley Online Library, 2108.

[17] V. Luboz, M. Bailet, C. Boichon Grivot, M. Rochette, B. Diot, M. Bucki, and Y. Payan, "Personalized modeling for real-time pressure ulcer prevention in sitting posture," Journal of Tissue Viability, vol. 27, pp. 54-58, Feb. 2018.

[18] M. I. Miga, K. Sun, I. Chen, L. W. Clements, T. S. Pheiffer, A. L. Simpson, and R. C. Thompson, "Clinical evaluation of a model-updated imageguidance approach to brain shift compensation: experience in 16 cases," International journal of computer assisted radiology and surgery, vol. 11, no. 8, pp. 1467-1474, 2015.

[19] S. Ji, X. Fan, A. Hartov, D. W. Roberts, and K. D. Paulsen, "Estimation of Intraoperative Brain Deformation," in Soft Tissue Biomechanical Modeling for Computer Assisted Surgery, pp. 97-133, Springer, 2012. 
[20] X. Fan, S. Ji, J. D. Olson, D. W. Roberts, A. Hartov, and K. D. Paulsen, "Image updating for brain deformation compensation in tumor resection," in Proc. SPIE Medical Imaging, vol. 9786, pp. 97862A-97862A-8, 2016.

[21] I. J. Gerard, M. Kersten-Oertel, S. Drouin, J. A. Hall, K. Petrecca, D. De Nigris, D. A. Di Giovanni, T. Arbel, and D. L. Collins, "Combining intraoperative ultrasound brain shift correction and augmented reality visualizations: a pilot study of eight cases," Journal of Medical Imaging, vol. 5, p. 1, Jan. 2018.

[22] X. Fan, D. W. Roberts, T. J. Schaewe, S. Ji, L. H. Holton, D. A. Simon, and K. D. Paulsen, "Intraoperative image updating for brain shift following dural opening," Journal of Neurosurgery, vol. 126, pp. 1924-1933, June 2017.

[23] M. Shakeri, E. Ferrante, S. Tsogkas, S. Lippe, S. Kadoury, I. Kokkinos, and N. Paragios, "Prior-based Coregistration and Cosegmentation," Medical Image Computing and Computer-Assisted Intervention - MICCAI 2016, vol. 2, pp. 529-537, 2016.

[24] O. M. Benkarim, G. Piella, M. A. Gonzalez Ballester, and G. Sanroma, "Enhanced Probabilistic Label Fusion by Estimating Label Confidences Through Discriminative Learning," Medical Image Computing and Computer-Assisted Intervention - MICCAI 2016, vol. 2, pp. 505-512, 2016.

[25] C. Arthofer, P. S. Morgan, and A. Pitiot, "Hierarchical Multi-Atlas Segmentation Using Label-Specific Embeddings, Target-Specific Templates and Patch Refinement," International Workshop on Patch-based Techniques in Medical Imaging, pp. 84-91, 2016.

[26] P. A. Yushkevich, J. Piven, H. Cody Hazlett, R. Gimpel Smith, S. Ho, J. C. Gee, and G. Gerig, "User-guided 3d active contour segmentation of anatomical structures: Significantly improved efficiency and reliability," NeuroImage, vol. 31, no. 3, pp. 1116-1128, 2006.

[27] CGAL, CGAL User and Reference Manual. CGAL Editorial Board, 4.9 ed., 2016.

[28] D. Lesage, E. D. Angelini, I. Bloch, and G. Funka-Lea, "A review of 3d vessel lumen segmentation techniques: Models, features and extraction schemes," Medical Image Analysis, vol. 13, pp. 819-845, 2009.

[29] M. Vermandel, N. Betrouni, C. Taschner, C. Vasseur, and J. Rousseau, "From MIP to MRA segmentation using fuzzy set theory," Computerized Medical Imaging and Graphics, vol. 31, pp. 128-140, 2007.

[30] F. Faure, C. Duriez, H. Delingette, J. Allard, B. Gilles, S. Marchesseau, H. Talbot, H. Courtecuisse, G. Bousquet, I. Peterlik, and S. Cotin, "SOFA: A Multi-Model Framework for Interactive Physical Simulation," in Soft 
Tissue Biomechanical Modeling for Computer Assisted Surgery, pp. 283321, Springer Berlin Heidelberg, 2012.

[31] I. Reinertsen, M. Descoteaux, K. Siddiqi, and D. L. Collins, "Validation of vessel-based registration for correction of brain-shift," Medical Image Analysis, vol. 11, pp. 374-388, 2007.

[32] A. Wittek, T. Hawkins, and K. Miller, "On the unimportance of constitutive models in computing brain deformation for image-guided surgery," Biomechanics and modeling in mechanobiology, vol. 8, no. 1, pp. 77-84, 2009 .

[33] P. Schiavone, F. Chassat, T. Boudou, E. Promayon, F. Valdivia, and Y. Payan, "In vivo measurement of human brain elasticity using a light aspiration device," Medical Image Analysis, vol. 13, pp. 673-678, 2009.

[34] M. Muller, J. Dorsey, L. McMillan, R. Jagnow, and B. Cutler, "Stable real-time deformations," Proceedings of ACM SIGGRAPH Symposium on Computer Animation (SCA), pp. 49-54, 2002.

[35] P. J. Besl and N. D. McKay, "A Method for Registration of 3-D Shapes," IEEE Transactions on Pattern Analysis and Machine Intelligence, vol. 14, no. 2, pp. 239-256, 1992.

[36] G. Saupin, C. Duriez, S. Cotin, and L. Grisoni, "Efficient Contact Modeling using Compliance Warping," in computer graphics international, (Istambul, Turkey), 2008.

[37] H. Courtecuisse, I. Peterlik, R. Trivisonne, C. Duriez, and S. Cotin, "Constraint-based simulation for non-rigid real-time registration," Medicine Meets Virtual Reality 21: NextMed/MMVR21, vol. 196, pp. 7682, 2014.

[38] I. Reinertsen, F. Lindseth, C. Askeland, D. H. Iversen, and G. Unsgard, "Intra-operative correction of brain-shift," Acta Neurochirurgica, vol. 156, pp. 1301-1310, 2014.

[39] A. Gronningstaeter, A. Kleven, S. Ommedal, T. E. Aarseth, T. Lie, F. Lindseth, T. Lango, and G. Unsgard, "SonoWand, an ultrasound-based neuronavigation system," Neurosurgery, vol. 47, no. 6, pp. 1373-1380, 2001.

[40] I. Reinertsen, F. Lindseth, G. Unsgard, and D. L. Collins, "Clinical validation of vessel-based registration for correction of brain-shift," Medical Image Analysis, vol. 11, pp. 673-684, 2007.

[41] C. Askeland, O. V. Solberg, J. B. L. Beate, I. Reinertsen, G. A. Tangen, E. F. Hofstad, D. H. Iversen, C. Vapenstad, T. Selbekk, T. Lango, T. A. Hernes, H. O. Leira, G. Unsgard, and F. Lindseth, "CustusX: an opensource research platform for image-guided therapy," International journal of computer assisted radiology and surgery, vol. 11, no. 4, pp. 505-519, 2015 . 
[42] F. Morin, H. Courtecuisse, I. Reinertsen, F. L. Lann, O. Palombi, Y. Payan, and M. Chabanas, "Resection-induced brain-shift compensation using vessel-based methods," in Proc. SPIE Medical Imaging 2018: Image-Guided Procedures, Robotic Interventions, and Modeling, vol. 10576, pp. 105760Q105760Q-6, 2018. 